\title{
Distribuição espacial e abundância das assembléias de peixes no Parque Nacional da Lagoa do Peixe, Rio Grande do Sul, Brasil
}

\author{
Daniel Loebmann ${ }^{1} \&$ João P. Vieira ${ }^{2}$ \\ ${ }^{1}$ Embrapa Meio-Norte. Rodovia BR-343, Km 35, Caixa Postal 341, 64200-970 Parnaíba, Piauí, Brasil. \\ E-mail: pinguimfiel@yahoo.com.br \\ ${ }^{2}$ Fundação Universidade Federal de Rio Grande. Caixa Postal 474, 96201-900 Rio Grande, Rio Grande do Sul, Brasil. \\ E-mail: docjpv@furg.br
}

\begin{abstract}
Spatial distribution and abundance of fish fauna at the Lagoa do Peixe National Park, Rio Grande do Sul, Brazil. This work investigates the spatial distribution and abundance of the ichthyofauna along the saline gradient of the Lagoa do Peixe National Park in southern Brazil. This system is an intermittent lagoon connected to the ocean by a single opening, which allows the mixing of salt and fresh water into the lagoon. As a consequence the ichthyofauna was composed of limnetic, marine and estuarine-related species. The unusual location of the opening of the estuary, near the center of the system, lead to an uncommon spatial distribution of fish fauna, which shows pre-limnetic characteristics at both the north and south zones and estuarine-marine affinities in the middle of the Lagoon. Based on fish species composition the lagoon could be separated into four sub-systems: pre-limnetic shallow-waters, pre-limnetic deep-waters, estuarine shallow-waters and estuarine deepwaters. The Fish composition was characterized by a gradual replacement of marine estuarine related species by fresh water species followed by changes in the dominance of selected species. At the shallow waters the subsystems also show a pronounced reduction in fish abundance from estuarine to fresh waters.
\end{abstract}

KEY WORDS. Estuary, ichthyofauna, conservation unit.

RESUMO. Este trabalho analisou a distribuição espacial e a abundância relativa da ictiofauna ao longo do gradiente salino do Parque Nacional da Lagoa do Peixe. Em função de apresentar uma barra intermitente, que permite a entrada de água salgada dentro de um sistema alimentado constantemente por água de baixa salinidade, proveniente de banhados e arroios, a Lagoa do Peixe apresentou uma ictiofauna composta de espécies límnicas, estuarino-relacionadas e marinhas. Baseado na composição específica da ictiofauna, a Lagoa pode ser compartimentada em quatro subsistemas: pré-límnico raso, pré-límnico profundo, estuarino raso e estuarino profundo. A variabilidade espacial na composição da ictiofauna revelou que existe uma mudança gradual na dominância das principais espécies, havendo uma substituição gradativa das espécies marinhas-estuarino relacionadas por peixes de água doce, e uma redução pronunciada nas abundâncias das espécies na zona rasa. A localização geográfica da barra, no centro da Lagoa, induz a uma divisão espacial ambiental atípica, quando comparados aos outros estuários do estado do Rio Grande do Sul, pois tanto para o Norte como para o Sul, há presença de uma zona pré-límnica. Este comportamento faz com que a Lagoa do Peixe apresente uma ictiofauna semelhante nos extremos Norte e Sul, separados por espécies estuarina e marinhas associadas às águas próximas a desembocadura central.

PALAVRAS CHAVE. Estuário, Ictiofauna, unidade de conservação.

O Parque Nacional da Lagoa do Peixe está localizado no segmento mediano da planície costeira do extremo sul do Brasil, entre a Lagoa dos Patos e o Oceano Atlântico. O Parque foi criado pelo Decreto-Lei $n^{\circ} 93.546$ em 1986 por sugestão do Instituto Brasileiro de Desenvolvimento Florestal - IBDF, hoje denominado Instituto Brasileiro do Meio Ambiente e Recursos Naturais Renováveis (IBAMA). Atualmente, o Parque possui status de Reserva da Biosfera, Sítio Ramsar e Reserva Internacional de
Aves Limnícolas. Dentro da área destaca-se como principal corpo d'água a Lagoa do Peixe, que é caracterizada como um ambiente lagunar devido sua comunicação intermitente com o mar. Embora já existam levantamentos preliminares da ictiofauna do Parque (de Bem Jr. \& Laurino 1994, Ramos \& Vieira 2001), aspectos relacionados com a estrutura da assembléia de peixes, como distribuição espacial e abundância relativa das espécies, não foram ainda considerados. 
A necessidade de obter informações científicas adicionais sobre a ictiofauna do Parque, aliada às dificuldades em estabelecer um esforço de coleta sistemático, em virtude de diversos fatores como distância dos grandes centros urbanos, alojamentos precários, difícil acesso e deslocamento, justificam por si só, o desenvolvimento deste trabalho. Além disso, as compreensões da ecologia dos organismos, sobretudo daqueles que apresentam seus ciclos de vida associados aos estuários, é fundamental para aprimorar o manejo e a conservação destes recursos renováveis.

\section{MATERIAL E MÉTODOS}

\section{Área de Estudo}

A Lagoa do Peixe (Fig. 1) está situada entre os pares de coordenadas geográficas $\left(31^{\circ} 26^{\prime} 37,3^{\prime \prime} \mathrm{S} ; 51^{\circ} 09^{\prime} 44,8^{\prime \prime} \mathrm{W}\right.$ e $31^{\circ} 14^{\prime}$ $\left.0,8^{\prime \prime} \mathrm{S} ; 51^{\circ} 00^{\prime} 49,2^{\prime \prime} \mathrm{W}\right)$. A Lagoa é classificada como um ambiente lagunar semi-fechado, onde, devido ao intercâmbio com seus sistemas vizinhos, ocorrem trocas e variações nas características físico-químicas da água. A barra da Lagoa apresenta comunicação intermitente com o Oceano Atlântico, e geralmente permanece fechada durante alguns meses do ano, acumulando água de banhados e lagoas adjacentes. Atingido determinado nível, a barra se rompe em direção ao mar (SCHWARZbold \& SCHÄFER 1984) e, quando isso não ocorre, a barra é aberta intencionalmente pelo homem. Esta Lagoa possui um espelho de água de aproximadamente $35 \mathrm{Km}^{2}$ e sua profundidade média é de $30 \mathrm{~cm}$, podendo chegar a $2 \mathrm{~m}$ nos canais e na barra.

A área de estudo, que compreende a porção estuarina e pré-limnica da Lagoa foi compartimentada em dez pontos, sendo quatro deles considerados como zona rasa (Barra, Manduca, Guaritas e Talha Mar) e seis de zona profunda (Chica, CapitãoRosa, Paiva, Lagamarzinho, Costa e Véia Terra). Um arroio tributário da Lagoa do Peixe, próximo da estrada que liga o balneário Praia-Nova à Mostardas ( $31^{\circ} 06^{\prime} 54,7^{\prime \prime}$ S; 5050'46,7"W), também foi amostrado e, em função de terem sido utilizados amostradores diferentes daqueles usados na Lagoa do Peixe, os dados ali obtidos foram somente considerados qualitativos.

\section{Desenho amostral}

Amostragens sazonais foram realizadas durante o inverno e primavera de 2001 e verão e outono de 2002. Em adição a estas coletas, durante o verão de 2002, houve intensificação das amostragens, onde foi possível realizar coletas mensais, além de incluir o rejeito da pesca do camarão-rosa com a rede tipo "Aviãozinho".

As coletas foram autorizadas pelo IBAMA, por meio da licença de $n^{\circ}$ 074/2001. O material coletado foi fixado em campo com formaldeído a $4 \%$ e triado nos Laboratórios da Fundação Universidade Federal do Rio Grande ou no Farol de Mostardas.

Foram utilizadas diversas artes de pesca para uma melhor descrição da ictiofauna. Os aparelhos de pesca utilizados foram: Picaré de arrasto-de-praia com $9 \mathrm{~m}$ de comprimento, com malha de $5 \mathrm{~mm}$ nos $3 \mathrm{~m}$ centrais e $12 \mathrm{~mm}$ nas laterais, com 1,5 m de altura, perfazendo uma área aproximada de $60 \mathrm{~m}^{2}$; Rede de cer- co com $20 \mathrm{~m}$ de comprimento com panagem única de $12 \mathrm{~mm}$, com 0,6 m de altura, varrendo uma área de aproximadamente $100 \mathrm{~m}^{2}$; Duas tarrafas de olho com abertura circular de $20 \mathrm{~m} \mathrm{e}$ malha de $12 \mathrm{~mm}$ e outra com abertura circular de $17 \mathrm{~m}$ e malha de $50 \mathrm{~mm}$. Uma tarrafa de rufo com abertura circular de $8 \mathrm{~m}$ e malha de $13 \mathrm{~mm}$; Rejeito da rede Aviãozinho utilizado por pescadores artesanais de camarão da região durante a noite por aproximadamente 12 horas, que consiste em um aparelho de pesca passivo com atrativo luminoso composta por três funis concêntricos com malha $12 \mathrm{~mm}$; Puçá com abertura semicircular de aproximadamente $128 \mathrm{~cm}$ com malha de $5 \mathrm{~mm}$ totalizando duas amostras e; Rede Quadrada com armação de cano (PVC) formando um quadrado de 0,8 m de lado, toda a panagem é de malha de $5 \mathrm{~mm}$ e a rede é arrastada com auxílio de um cabo, varrendo aproximadamente $8 \mathrm{~m}^{2}$. O número de amostras realizadas com cada arte de pesca para cada setor está descrito na tabela I.

Tabela I - Esforço de pesca (número de amostras) aplicado para cada setor amostrado durante o estudo. (CER) Rede-de-cerco, (PIC) Rede picaré, (TRF) Tarrafa, (RQU) Rede quadrada, (AVI) aviãozinho.

\begin{tabular}{lcccccc}
\hline \multicolumn{1}{c}{ Setor } & CER & PIC & TRF & PUÇ & RQU & AVI \\
\hline Manduca & 17 & 30 & 4 & & & \\
Chica & & & & & & 4 \\
Capitão rosa & & & & & & 6 \\
Barra & 20 & 40 & 7 & & & \\
Guaritas & & 27 & 2 & 1 & 2 & \\
Paiva & & & & & & 8 \\
Lagamarzinho & & & & & & 11 \\
Costa & & & & & & 10 \\
Talha mar & 1 & 29 & 7 & & & \\
Véia terra & & & & & & 9 \\
Ponte norte & & 3 & 6 & 1 & 13 & \\
\hline
\end{tabular}

\section{Análise dos dados}

A Lagoa do Peixe foi subdividida por profundidade, em zona rasa $(<1 \mathrm{~m})$ e zona profunda (entre 1 e $2 \mathrm{~m}$ ) para as análises quantitativas. Para a zona rasa foram consideradas apenas as amostras provenientes da rede picaré (setores Manduca, Barra, Guaritas e Talha Mar) e para as zonas profundas as amostras provenientes das redes de aviãozinho (setores Chica, Capitão Rosa, Paiva, Lagamarzinho, Costa e Véia Terra).

Para fins comparativos aplicou-se o índice de captura por unidade de esforço (CPUE), com o propósito de observar possíveis mudanças de espécies dominantes ao longo da Lagoa. Em função dos dados de fundo e raso serem oriundos de amostradores diferentes, as comparações de capturas não puderam ser realizadas entre assembléias de fundo e raso, ou seja, os dados quantitativos só foram comparáveis entre setores que utilizaram os mesmos amostradores. 


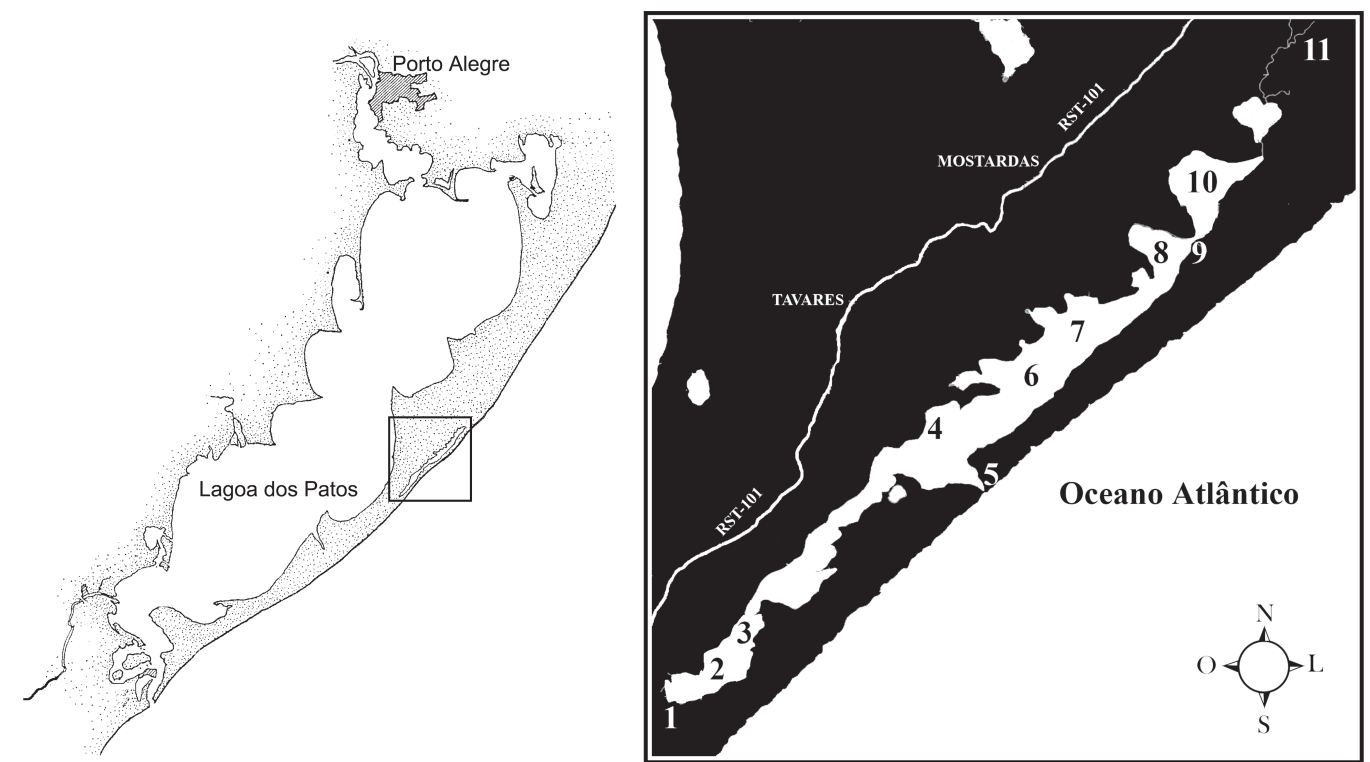

Figura 1. Área de estudo com seus respectivos pontos de coleta. (1) Manduca, (2) Chica, (3) Capitão-Rosa, (4) Guaritas, (5) Barra, (6) Paiva, (7) Lagamarzinho, (8) Costa, (9) Talha-Mar, (10) Veia-Terra, (11) Ponte Norte.

Para cada espécie, foi calculado o grau de importância relativa em cada amostrador através da freqüência de ocorrência percentual (FO\%) e percentagem numérica (PN\%). Onde as espécies foram classificadas em: espécies ocasionais que não ultrapassaram valores acima da média de PN\% e FO\%; espécies freqüentes e não abundantes, com valores de PN\% menor que a média de PN\% e valores FO\% acima da média de FO\%; espécies freqüentes e abundantes, com valores de PN\% e FO\% acima da média.

Segundo Vieira et al. (1996) calculou-se o índice de importância relativa $(\mathrm{IIR} \%=\mathrm{FO} \% \mathrm{x}(\mathrm{PN} \%+\mathrm{PW} \%))$, que leva em consideração a freqüência de ocorrência percentual (FO\%), a abundância relativa numérica percentual (PN\%) baseada no valor da CPUE em número, e a biomassa relativa percentual (PW\%), baseada na CPUE em peso. Com base nos valores do IIR\% aplicou-se uma análise de agrupamento, com o propósito de agrupar os locais de coleta quanto à sua semelhança. $\mathrm{O}$ método Complete Linkage foi empregado, com distâncias calculadas com base em City-Block (Manhattan distances).

\section{RESULTADOS}

A utilização dos seis aparelhos de coleta, nos 11 setores da amostrados (10 setores da Lagoa do Peixe mais o arroio tributário Ponte Norte) resultou na coleta de 53.968 peixes pertencentes a 25 famílias e 67 espécies (Fig. 2). No setor CapitãoRosa foi registrado o menor número de espécies $(S=12)$ e no setor Ponte Norte o maior número $(S=26)$, este último favorecido pelo grande número de espécies límnicas como Astyanax jacuhiensis, Astyanax fasciatus, Callichthys callichthys, Cheirodon ibicuhiensis, Mimagoniates inequalis, Pseudocorynopoma doriae, Cyphocarax saladensis e Eigenmannia virescens exclusivas deste setor. Exemplares de Jenynsia multidentata foram coletadas em todos os 11 setores, e as espécies Atherinella brasiliensis, Gobionellus oceanicus, Platanichthys platana, Odontesthes argentinensis também apresentaram ampla distribuição ao longo da Lagoa.

De modo geral, as distribuições espaciais das espécies coletadas (Fig. 2) apresentam três padrões, sendo que o grupo das espécies límnicas apresentou $\mathrm{S}=32$ com tendência a se distribuir nos extremos Norte e Sul da Laguna; o grupo das espécies estuarino-relacionadas apresentou $S=16$, com maior amplitude espacial, sendo algumas espécies encontradas em toda extensão da Lagoa; o grupo das espécies marinhas apresentou uma riqueza de espécies intermediária sendo $S=19$, tendendo a se concentrar na região central da Lagoa.

Os resultados das análises quantitativas foram representados por 47.805 indivíduos e 56 espécies (13.957 indivíduos e 46 espécies capturados na pesca com aviãozinho e 33.848 indivíduos e 33 espécies capturados na pesca com Picaré). Na zona rasa Mugil platanus foi a espécie mais abundante em número, compreendendo $76,4 \%$ dos indivíduos capturados, seguida de J. multidentata (9,3\%), Astyanax eingenmaniorum (4,4\%), O. argentinensis $(2,9 \%)$ e $A$. brasiliensis $(1,9 \%)$. Juntas estas espécies perfizeram $94,9 \%$ do número total de peixes capturados nas zonas rasas (Fig. 3). Na zona profunda, Brevoortia pectinata foi a espécie mais abundante em número, compreendendo 42,5\% dos indivíduos capturados seguidas por Micropogonias furnieri (16,7\%), J. multidentata (14,6\%), Geophagus brasiliensis $(7,6 \%)$, O. argentinensis (5,3\%), Eucinostomus argenteus (3,7\%) е $M$. platanus $(2,6 \%)$, que totalizam $93,8 \%$ do número total de peixes capturados nesta zona (Fig. 4).

Revista Brasileira de Zoologia 22 (3): 667-675, setembro 2005 


\begin{tabular}{|c|c|c|c|c|c|c|c|c|c|c|c|c|c|}
\hline \multirow{2}{*}{ ORIGEM } & \multirow{2}{*}{ FAMÍLIA } & \multirow{2}{*}{ NOME CIENTÍFICO } & \multicolumn{3}{|c|}{$\mathrm{ZPL}$} & \multicolumn{5}{|c|}{$\overline{Z E}$} & \multicolumn{2}{|c|}{$\overline{\mathrm{ZPL}}$} & \multirow{2}{*}{\begin{tabular}{|l|}
$\mathrm{ZL}$ \\
$\mathrm{PON}$ \\
\end{tabular}} \\
\hline & & & \begin{tabular}{|l|l|l|l} 
MAN \\
\end{tabular} & $\mathrm{CHI}$ & CAP & GUA & BAR & PAl & LAG & $\cos$ & TAL & DEI & \\
\hline \multirow{16}{*}{$\begin{array}{l}E \\
S \\
P \\
\text { E } \\
C \\
I \\
E \\
S\end{array}$} & Cichlidae & Geophagus brasiliensis (Quoy \& Gaimard, 1824) & & & & & & & & & & & \\
\hline & Characidae & Astyanax eingemanniorum (Cope, 1894) & & & & & & & & & & & \\
\hline & Characidae & Hyphessobrycon igneus (Miquelarena, Menni, Lopez \& Casciotta, 1980) & & & & & & & & & & & \\
\hline & Characidae & Oligosarcus jenynsii (Günther, 1864) & & & & & & & & & & & \\
\hline & Callichthydae & Corydoras paleatus (Jenyns, 1842) & & & & & & & & & & & \\
\hline & Curimatidae & Cyphocarax voga (Hensel, 1870) & & & & & & & & & & & \\
\hline & Poeciliidae & Phalloceros caudimaculatus (Hensel, 1868) & & & & & & & & & & & \\
\hline & Characidae & Hyphessobrycon luetkenii (Boulenger, 1887) & & & & & & & & & & & \\
\hline & Cichlidae & Cichlasoma portalegrense (Hensel, 1870) & & & & & & & & & & & \\
\hline & Erythrinidae & Hoplias malabaricus (Bloch,1794) & & & & & & & & & & & \\
\hline & Pimelodidae & Pimelodella australis Eigenmann, 1917 & & & & & & & & & & & \\
\hline & Poeciliidae & Phalloptychus januarius (Hensel, 1868) & & & & & & & & & & & \\
\hline & Characidae & Cheirodon interruptus (Jenyns, 1842) & & & & & & & & & & & \\
\hline & Cichlidae & Crenicichla lepidota Heckel, 1840 & & & & & & & & & & & \\
\hline & Pimelodidae & Rhamdia quelen (Quoy \& Gaimard, 1824) & & & & & & & & & & & \\
\hline & \begin{tabular}{|l|ll} 
Callichthydae \\
\end{tabular} & Hoplosternum littorale (Hancock, 1828) & & & & & & & & & & & \\
\hline L & \begin{tabular}{|l|} 
Characidae \\
\end{tabular} & Hyphessobrycon boulengeri (Eigenmann, 1907) & & & & & & & & & & & \\
\hline i & \begin{tabular}{|l|l} 
Callichthydae \\
\end{tabular} & Callichthys callichthys (Linnaeus, 1758) & & & & & & & & & & & \\
\hline M & Characidae & Cianocharax alburnus (Hensel, 1870) & & & & & & & & & & & \\
\hline $\mathrm{N}$ & Characidae & Astyanax jacuhiensis Cope, 1894 & & & & & & & & & & & \\
\hline 1 & Characidae & Astyanax fasciatus (Cuvier, 1819) & & & & & & & & & & & \\
\hline c & Characidae & Characidium rachovii Regan, 1913 & & & & & & & & & & & \\
\hline A & \begin{tabular}{|l|} 
Characidae \\
\end{tabular} & Cheirodon ibicuhiensis Eingenmann, 1915 & & & & & & & & & & & \\
\hline s & Characidae & Hyphessobrycon meridionalis Ringuelet, Miquelarena \& Menni, 1978 & & & & & & & & & & & \\
\hline & Characidae & Mimagoniates inequalis (Eigenmann, 1915) & & & & & & & & & & & \\
\hline & Characidae & Oligosarcus robustus Menezes, 1969 & & & & & & & & & & & \\
\hline & Characidae & Pseudocorynopoma doriae Perugia, 1891 & & & & & & & & & & & \\
\hline & Cichlidae & Cichlasoma facetum (Jenyns, 1842) & & & & & & & & & & & \\
\hline & Curimatidae & Cyphocarax saladensis (Meinken, 1933) & & & & & & & & & & & \\
\hline & Eleotridae & Dormitator maculatus (Bloch, 1792) & & & & & & & & & & & \\
\hline & Sternopygidae & Eigenmannia virescens (Valenciennes, 1842) & & & & & & & & & & & \\
\hline & Poeciliidae & Cnesterodon decemmaculatus (Jenyns, 1842) & & & & & & & & & & & \\
\hline & Gobiidae & Awaous tajasica (Lichtenstein, 1822) & & & & & & & & & & & \\
\hline & Mugilidae & Mugil gaimardianus Desmarest, 1831 & & & & & & & & & & & \\
\hline & Bothidae & Paralichthys orbignyanus Valenciennes, 1839 & & & & & & & & & & & \\
\hline & Centropomidae & Centropomus parallelus (Poey, 1860) & & & & & & & & & & & \\
\hline ESL L & Engraulidae & Lycengraulis grossidens (Agassiz, 1829) & & & & & & & & & & & \\
\hline ST A & Mugilidae & Mugil curema Valenciennes, 1836 & & & & & & & & & & & \\
\hline$P \cup C$ & Mugilidae & Mugil platanus Gunther, 1880 & & & & & & & & & & & \\
\hline É A I & Sciaenidae & Micropogonias furnieri (Desmarest, 1823) & & & & & & & & & & & \\
\hline $\mathrm{CRO}$ & Eliotridae & Eleotris pisonis (Gmelin, 1789) & & & & & & & & & & & \\
\hline I I N & Ariidae & Genidens genidens (Valenciennes, 1839) & & & & & & & & & & & \\
\hline EN A & Gobiidae & Gobionellus shufeldti (Jordan \& Gilbert, 1887) & & & & & & & & & & & \\
\hline$S A D$ & Atherinidae & Odontesthes argentinensis (Valenciennes, 1835) & & & & & & & & & & & \\
\hline S A & Clupeidae & Platanichthys platana (Regan, 1917) & & & & & & & & & & & \\
\hline s & Gobiidae & Gobionellus oceanicus (Pallas, 1770) & & & & & & & & & & & \\
\hline & Atherinidae & Atherinella brasiliensis (Quoy \& Gaimard, 1824) & & & & & & & & & & & \\
\hline & Jenynsiidae & Jenynsia multidentata (Jenyns, 1842) & & & & & & & & & & & \\
\hline & Clupeidae & Brevoortia pectinata (Jenyns, 1842) & & & & & & & & & & & \\
\hline 1 & Gerreidae & Eucinostomus argenteus Baird \& Girard 1855 & & & & & & & & & & & \\
\hline s & \begin{tabular}{|l|} 
Bothidae \\
\end{tabular} & Citharichthys spilopterus Günther, 1862 & & & & & & & & & & & \\
\hline 1 & \begin{tabular}{|l|} 
Carangidae \\
\end{tabular} & Trachinotus carolinus (Linnaeus, 1766) & & & & & & & & & & & \\
\hline $\mathrm{T}$ & Carangidae & Trachinotus marginatus Cuvier, 1832 & & & & & & & & & & & \\
\hline A & Carangidae & Selene vomer (Linnaeus, 1758) & & & & & & & & & & & \\
\hline $\mathrm{N}$ & Serranidae & Epinephelus marginatus (Lowe, 1834) & & & & & & & & & & & \\
\hline $\mathrm{T}$ & Clupeidae & Harengula clupeola (Cuvier, 1829) & & & & & & & & & & & \\
\hline E & Elopidae & Elops saurus Linnaeus, 1766 & & & & & & & & & & & \\
\hline $\mathrm{s}$ & Pomatomidae & Pomatomus saltatrix (Linnaeus, 1766) & & & & & & & & & & & \\
\hline & Carangidae & Uraspis secunda (Poey, 1860) & & & & & & & & & & & \\
\hline M & Clupeidae & Sardinella aurita Valenciennes, 1847 & & & & & & & & & & & \\
\hline A & Elopidae & Albula nemoptera (Fowler, 1911) & & & & & & & & & & & \\
\hline $\mathrm{R}$ & Engraulidae & Anchoa marinii (Hildebrand, 1943) & & & & & & & & & & & \\
\hline N & Gadidae & Urophycis brasiliensis (Kaup, 1858) & & & & & & & & & & & \\
\hline $\mathrm{N}$ & Sciaenidae & Pogonias cromis (Linnaeus, 1766) & & & & & & & & & & & \\
\hline $\begin{array}{l}\mathrm{H} \\
0\end{array}$ & Sciaenidae & Scienidae não identificado & & & & & & & & & & & \\
\hline s & Sciaenidae & Stellifer brasiliensis (Schultz) & & & & & & & & & & & \\
\hline 8 & Serranidae & Mycteroperca acutirostris (Valenciennes, 1828) & & & & & & & & & & & \\
\hline
\end{tabular}

Figura 2. Padrão de distribuição das espécies encontradas na Lagoa do Peixe. Obs: As estações de coleta foram dispostas do extremo sul da Lagoa do Peixe (Manduca) até o extremo norte (Ponte Norte) para evidenciar o padrão de distribuição. (ZPL) Zona pré-limnica, (ZE) zona estuarina e (ZL) zona límnica. (MAN) Manduca, (CHI) Chica, (CAP) Capitão-Rosa, (GUA) Guaritas, (BAR) Barra, (PAI) Paiva, LAG = Lagamarzinho, (COS) Costa, (TAL) Talha-Mar, (VEI) Veia-Terra, (PON) Ponte Norte.

Revista Brasileira de Zoologia 22 (3): 667-675, setembro 2005 

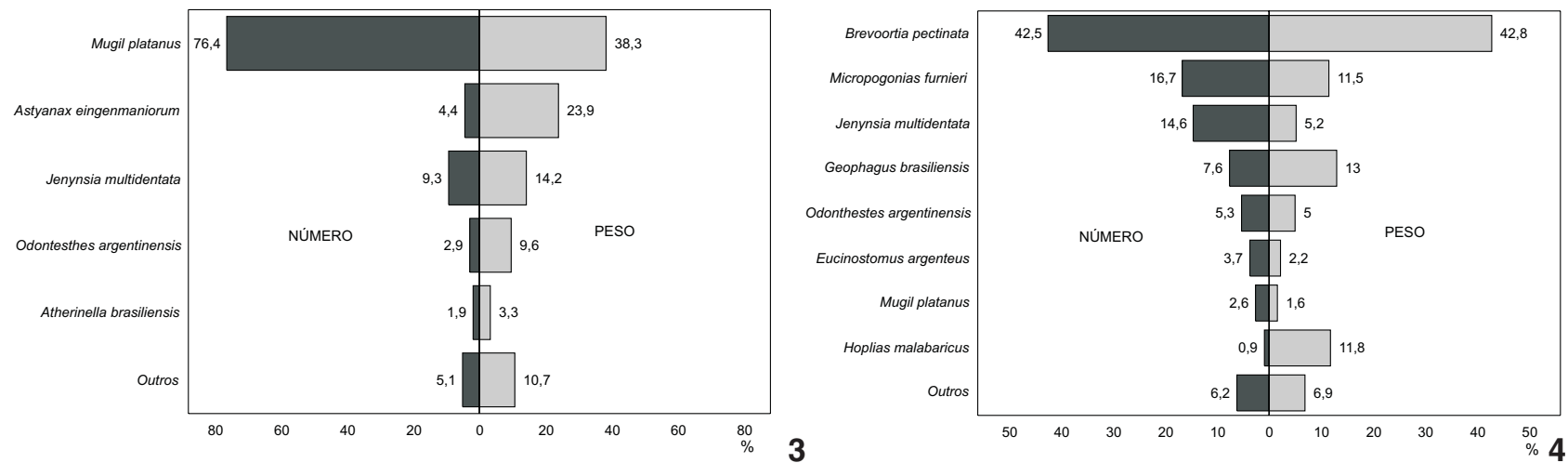

Figuras 3-4. Abundância percentual e biomassa percentual para as espécies mais importantes em todos os setores da zona rasa (3) e da zona profunda (4) da Lagoa do Peixe.

Com relação à biomassa capturada, um total de $157.377 \mathrm{~g}$ de peixes foram coletados, sendo $143.462 \mathrm{~g}$ provenientes da pesca com aviãozinho e $13.915 \mathrm{~g}$ nas amostragens com Picaré. Na zona rasa, $M$. platanus representou $38,3 \%$ do total capturado, $A$. eingenmaniorum contribuiu com $23,9 \%$ da captura total, $J$. multidentata representou 14,2\%, O. argentinensis 9,6\% e A. brasiliensis 3,3\%. Todas estas espécies somadas representaram $89,3 \%$ da biomassa total de peixes capturados nesta zona (Fig. 3). Na zona profunda, B. pectinata foi a espécie de maior biomassa, compreendendo $42,8 \%$ do peso total capturado. As espécies, Geophagus brasiliensis (13\%), Hoplias malabaricus (11,8\%), M. furnieri $(11,5 \%)$, J. multidentata $(5,2 \%)$, O. argentinensis (5\%), E. argenteus $(2,2 \%)$ e $M$. platanus $(1,6 \%)$ também foram importantes, perfazendo um total de $93,1 \%$ do peso total capturado na zona profunda da Lagoa do Peixe (Fig. 4).

A análise de agrupamento, baseada no Índice de Importância Relativa, agrupou os 10 setores estudados (Fig. 5) em quatro grandes zonas: zona pré-límnica rasa, zona pré-límnica profunda, zona estuarina rasa e zona estuarina profunda. As duas primeiras zonas foram representadas pelos setores dos extremos norte (Talha Mar, Véia Terra) e sul (Manduca, Chica e Capitão Rosa) da Lagoa, e as duas últimas representadas pelos setores centrais da Lagoa próximos à desembocadura (Barra e Guaritas para o raso; Paiva, Lagamarzinho e Costa, para o profundo).

Oligosarcus jenynsii, G. brasiliensis, $H$. malabaricus foram as espécies encontradas em maior freqüência, peso e número na zona pré-límnica profunda. Astyanax eingenmaniorum, $B$. pectinata, $J$. multidentata e $P$. platana também foram abundantes e freqüentes nesta zona. Desta forma, observa-se que, com a exceção de $P$. platana e $B$. pectinata, as espécies mais importantes são tipicamente límnicas. Na zona pré-límnica rasa, $A$. eingenmaniorum, G. brasiliensis, J. multidentata, M. platanus e $O$. jenysii foram as espécies de maior importância, ou seja, com exceção de $M$. platanus, a composição específica das espécies mais abundantes e freqüentes foi semelhante tanto para o raso, como para o fundo (Tab. II).

$\mathrm{Na}$ zona estuarina rasa, a alta abundância específica de

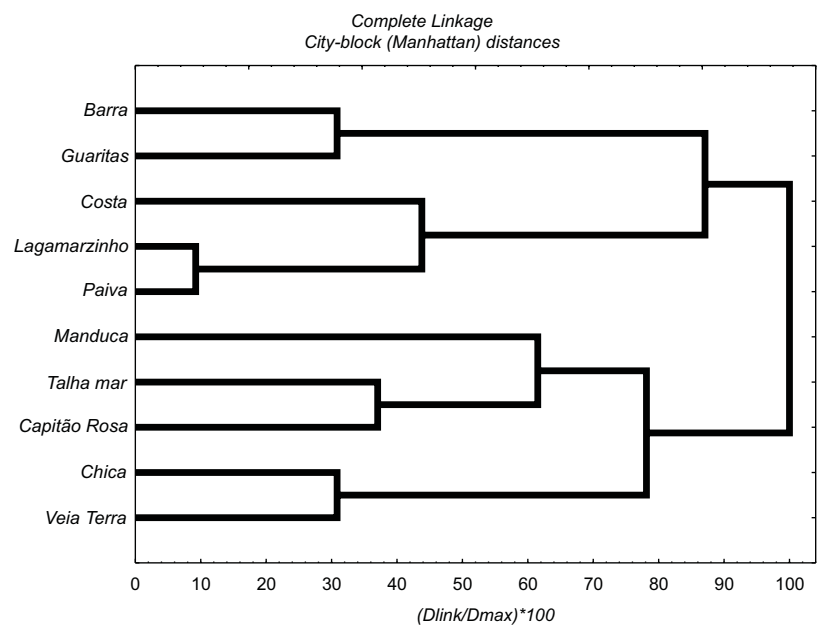

Figura 5. Análise de agrupamento baseada índice de importância relativa para os setores da Lagoa do Peixe (vide figuras 1 e 2 para caracterização dos pontos de coleta).

M. platanus fez esta espécie ser a única com valores acima da média neste critério de corte. No entanto, as espécies $J$. multidentata e $O$. argentinensis foram importantes em peso e estiveram presentes em aproximadamente $50 \%$ das amostras. Ou seja, este setor tem como característica marcante, poucas espécies abundantes e freqüentes, com predomínio de espécies estuarinorelacionadas. A zona estuarina profunda foi representada principalmente pelas espécies B. pectinata, E. argenteus, J. multidentata, M. furnieri e O. argentinensis.

Embora não comparáveis entre si devido ao uso de duas artes de pesca para os cálculos abaixo descritos, a Captura por Unidade de Esforço de cada setor (Tab. II) mostrou que a zonas rasas apresentram uma CPUE maior em relação a zonas profundas, influenciadas principalmente pelas elevadas capturas de $M$. platanus na zona rasa estuarina (CPUE $=381,76$ ind $/$ rede), enquanto que na zona profunda, $B$. pectinata apresentou os

Revista Brasileira de Zoologia 22 (3): 667-675, setembro 2005 


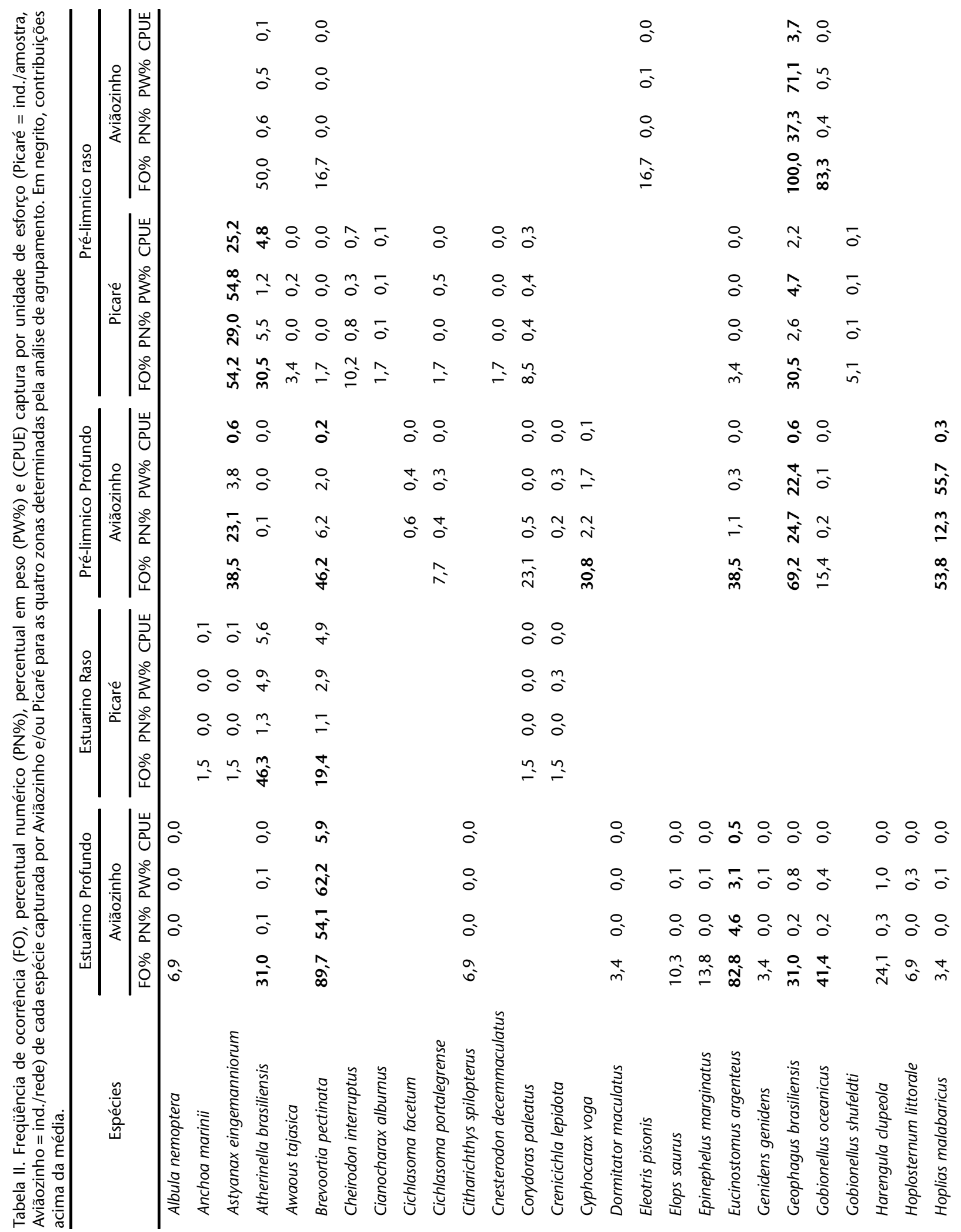




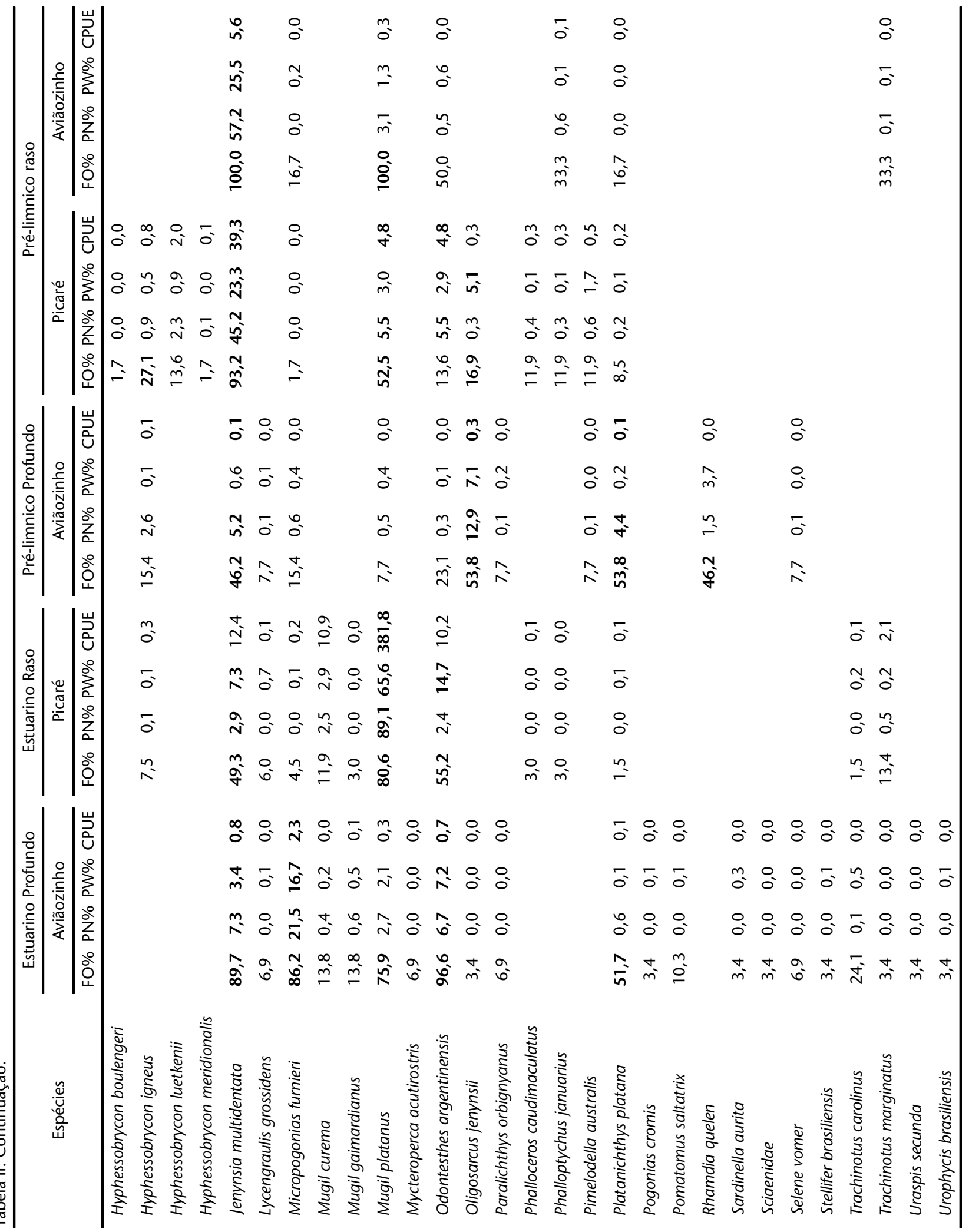


maiores valores de captura por unidade de esforço na zona central (CPUE $=5,88$ ind/rede) e, as regiões mais afastadas da desembocadura apresentaram diminuição da CPUE e substituição das espécies, sendo que espécies de origem límnica ( $A$. eingenmaniorum, G. brasiliensis) e estuarino-relacionadas ( $J$. multidentata) passaram a ter maiores capturas.

\section{DISCUSSÃO}

O presente estudo descreve as associações de peixes das zonas rasas e profundas da Lagoa do Peixe. A análise dos dados possibilitou a divisão ecológica deste ecossistema em dois grandes compartimentos, zona pré-límnica e zona estuarina, que por sua vez foram divididos, em função da profundidade relativa, em zonas rasas e zonas profundas. Ressalta-se que o setor Ponte Norte, embora não incluído na análise quantitativa, deve ser considerado uma associação à parte, que em função da predominância de espécies de peixes de água doce deve ser classificado como zona límnica.

Segundo KenNish (1990), a composição específica das populações de peixes estuarinos muda constantemente e drasticamente em razão da variabilidade das condições ambientais e dos limites específicos de tolerância de determinadas espécies às alterações no ambiente. A presença dos três grandes grupos de espécies, as de origem límnica, estuarina e marinha, é decorrente da característica da Lagoa do Peixe de apresentar, uma conexão com o oceano na zona central do sistema, onde, foram registrados elevados valores de salinidade $(\mathrm{S}=34)$ e, seus extremos norte e sul associados a arroios e banhados, que contribuem com uma massa de água de baixa salinidade $(<0,1)$. Esta estratificação espacial evidentemente resultou em uma diferenciação na composição ictiofaunística ao longo da Lagoa. Os extremos norte e sul apresentam uma ictiofauna com a maioria das espécies de origem límnica, e as espécies de origem marinha e estuarina concentraram-se no centro da Lagoa. Observou-se também, que a ictiofauna capturada nos extremos norte e sul, em geral, é representada por indivíduos adultos, enquanto que a zona central foi representada principalmente por juvenis.

O número de famílias que contribuem para a composição da fauna de peixes estuarinos geralmente é pequeno, uma vez que poucas espécies podem sobreviver nestes ambientes (Haedrich 1983, Vieira et al. 1996). No caso da Lagoa do Peixe, das 26 famílias encontradas, 23 estavam presentes na zona estuarina, reflexo de muitas espécies ocasionais ali encontradas, em função do agrupamento dos dados coletados ao longo de um ano. A variabilidade de espécies ocasionais em estuários está ligada às mudanças bruscas das características físico-químicas da água. Por esta razão a maioria das espécies de peixes que são encontrados em estuários ali permanecem por um curto período de tempo, como fazem os peixes de água doce, peixes diadrômicos, peixes marinhos não-dependentes e peixes marinhos dependentes (Moyle \& CECH 1988).

As zonas rasas dos estuários abrigam o maior contingente de peixes tipicamente estuarinos (DAY et al. 1989, VIEIRA \&
Musick 1994). Nelas vivem peixes de pequeno porte, adultos geralmente com menos de $10 \mathrm{~cm}$ ou juvenis de espécies marinhas estuarino relacionadas, constituídas principalmente pelas famílias Atherinidae, Mugilidae, Gerreidae, Cyprinodontidae e Anablepidae (VIEIRA \& Musick 1993). Confirmando este padrão a zona estuarina rasa da Lagoa do Peixe foi dominada por dois atherinídeos (Atherinella brasiliensis e Odontesthes argentinensis), um mugilídeo (Mugil platanus) e um anablepídeo (Jenynsia multidentata).

Na Lagoa do Peixe, em função de sua baixa profundidade média $(30 \mathrm{~cm})$ e a presença esparsa de canais relativamente rasos $(<2 \mathrm{~m})$ a associação de peixes da zona profunda é composta por uma mistura de espécies de meia-água (B. pectinata), raso (J. multidentata) e fundo (M. furnieri), associadas a presença do cará $G$. brasiliensis, uma espécie secundária de água doce capaz de tolerar altas salinidades (ANDreata et al. 1989, GarCia \& VIEIRA 1997).

Os grandes estuários apresentam um forte padrão longitudinal na taxa de troca de espécies desde a região de influência marinha até a região límnica (Weinstein et al. 1980, Peterson \& Ross 1991, Winemiller \& Leslie 1992, Wagner \& Austin 1999). O estuário da Lagoa do Peixe, apesar de sua pequena proporção $\left(35 \mathrm{Km}^{2}\right)$ também apresentou um nítido gradiente de espécies entre a porção central da laguna até os setores norte e sul, com a substituição gradativa de espécies marinhas por espécies de água doce.

O menor número de espécies estuarino-relacionadas $(\mathrm{S}=$ 16), sendo apenas $J$. multidentata e $A$. brasiliensis consideradas estuarino-residentes (Rozas \& Hackney 1984, Vieira \& Musick 1993), é explicado pelo fato de que poucos animais conseguem desenvolver mecanismos fisiológicos complexos suficientes para viver em ambientes que apresentam grandes variações físicas como os ambientes de águas salobras.

Em geral, as espécies da zona estuarina rasa foram de tamanho pequeno, em torno de 20 a $30 \mathrm{~mm}$, sendo que a grande maioria dos indivíduos era juvenil, como M. platanus. Esses dados são semelhantes aos encontrados para a zona estuarina do estuário da Lagoa dos Patos (GARCIA et al. 2001), caracterizando os estuários como zonas de criação para diversas espécies de peixes (HaEdrich 1983, Vieira et al. 1996).

Como conclusão, devemos salientar que a disposição da barra no centro da Lagoa do Peixe promove uma divisão espacial da Lagoa atípica, quando comparados aos outros estuários do Rio Grande do Sul, pois tanto para o norte como para o sul, há presença de uma zona pré-límnica. Este comportamento faz com que a Lagoa do Peixe apresente uma ictiofauna semelhante nos extremos norte e sul, separados por água de alta salinidade dos setores centrais.

\section{AGRADECIMENTOS}

Agradecemos a equipe do laboratório de Ictiologia da FURG que participaram ativamente nas atividades de campo e triagem do material. Ao IBAMA de Mostardas pelo apoio no trabalho de 
campo. A Marinha do Brasil pelo comodato da FURG junto ao Farol de Mostardas. Aos Drs. Luiz Roberto Malabarba e Carlos Alberto Santos de Lucena pela ajuda na identificação da ictiofauna límnica. À CAPES pela concessão de uma Bolsa de estudo (nível mestrado) para o primeiro autor. Aos Drs. Carlos Emílio Bemvenuti, Érica Maria Pellegrini Caramaschi, José Vanderli Andreata e Paulo de Tarso da Cunha Chaves pelas valiosas criticas e sugestões na versão original deste manuscrito.

\section{REFERÊNCIAS BIBLIOGRÁFICAS}

Andreata, J.V.; A.M. SAAD \& L.R.R. BARbieri. 1989. Associação e distribuição das espécies de peixes na Laguna de Marapendi, Rio de Janeiro, no período de marco de 1985 a fevereiro de 1987. Memórias do Instituto Oswaldo Cruz, Rio de Janeiro, 84 (4): 45-51.

DaY, J.W.; C.A.S. Hall; W.M. Kemp \& A. Yanez-Arancibia. 1989. Estuarine ecology. New York, John Wiley and Sons, 558p.

DE BEM JR., R.T. \& L.B. LAURINO. 1994. Contribuição ao conhecimento da ictiofauna da região do Parque Nacional da Lagoa do Peixe (RS) Brasil. Revista da UCPEL, Pelotas, 4 (2): 61-66.

Garcia, A.M. \& J.P. Vieira. 1997. Abundância e diversidade da assembléia de peixes dentro e fora de uma pradaria de Ruppia maritima L., no estuário da Lagoa dos Patos (RS-Brasil). Revista Atlântica, Rio Grande, 19: 161-181.

Garcia, A.M.; J.P. Vieira \& K.O. Winemiller. 2001. Dynamics of the shallow-water fish assemblage of the Patos Lagoon estuary (Brazil) during cold and warm ENSO episodes. Journal of Fish Biology, London, 59: 1218-1238.

Haedrich, R.L. 1983. Estuarine Fishes, p. 183-207. In: B.H. Kетсним (Ed.). Estuaries and Enclosed Seas. New York, Elsevier Scientific, vol. 26, 500p.

Kennish, M.J. 1990. Ecology of estuaries. Boca Raton, CRC Press, II+ 391p.

Moyle, P.B. \& J.J. СесH. 1988. Feeding, nutrition, digestion and excretion, p. 85-147. In: P.B. Moyle \& J.J. CECH (Eds). Fishes:
An introduction to ichthyology. New Jersey, Prentice Hall, Englewood Cliffs, 559p.

Peterson, M.S. \& S.T. Ross. 1991. Dynamics of littoral fishes and decapods along a coastal river-estuarine gradient. Estuarine, Coastal and Shelf Science, London, 33: 467-483.

Ramos, L. \& J.P. Vieira. 2001. Composição Específica e abundância de peixes de zonas rasas dos cinco estuários do Rio Grande do Sul, Brasil. Boletim do Instituto de Pesca, São Paulo, 27 (1): 109-121.

RozAS, L.P. \& C.T. HACKNEY 1984. Use of oligohaline marshes by fishes and macrofaunal crustaceans in North Carolina. Estuaries, Mississippi, 7 (3): 213-224.

Schwarzbold, A. \& A. SchÄFer 1984. Gênese das lagoas costeiras do Rio Grande do Sul. Amazoniana, Manaus, 9 (1): 84-104.

VieIRA, J.P. \& J.A. Musick. 1993. Latitudinal patterns in diversity of fishes in warm-temperate and tropical estuarine waters of the western Atlantic. Revista Atlântica, Rio Grande, 15: 115-133.

VieIRA, J.P. \& J.A. Musick. 1994. Fish fauna composition in warmtemperate and tropical estuaries of western Atlantic. Revista Atlântica, Rio Grande. 16: 31-53.

Vieira, J.P.; M.C. Vasconcellos; R.E. Silva \& L.C. Fisher. 1996. A rejeição da pesca Camarão-rosa (Penaeus paulensis) no estuário da Lagoa dos Patos, RS, Brasil. Revista Atlântica, Rio Grande, 18: 123-142.

Wagner, C.M. \& H.M. Austin. 1999. Correspondence between environmental gradients and summer littoral fish assemblages in low salinity reaches of the Chesapeake Bay, USA. Marine Ecology Progress Series, Nordbünte, 177: 197-212.

Weinstein, M.P.; S.L. Weiss \& M.F. WALTERS. 1980. Multiple determinants of community structure in shallow marsh habitats, Cape Fear river estuary, North Carolina, USA. Marine Biology, Heidelberg, 58: 227-243.

Winemiller, K.O. \& M.A. LesLie. 1992. Fish assemblages across a complex, tropical freshwater/marine ecotone. Environmental Biology of Fishes. Ontario, 34: 29-50.

Recebido em 14.X.2004; aceito em 12.VIII.2005. 